\title{
Rom e Sinti: problemi, tensioni e risorse per una programmazione strategica di interventi sociali.
}

\author{
di Tommaso Vitale e Loris Caruso
}

\section{Il mistero rom: la varietà}

Gli interventi rivolti alla minoranza rom e sinta sono fortemente marcati da una scarsa conoscenza dei gruppi in questione, se non dalla presenza di informazioni errate, di stereotipi radicati e di forti pregiudizi. Non entreremo qui nel merito della fenomenologia degli stereotipi diffusi nella popolazione italiana o fra gli operatori sociali (cfr. Vitale, Claps, Arrigoni, 2009). Cercheremo semmai di definire alcuni caratteri di questi gruppi la cui conoscenza è essenziale ai fini di una corretta programmazione sociale. Procederemo con estrema sintesi e per punti.

I gruppi zigani sono molti e assai eterogenei. Sono presenti in ogni Stato europeo da almeno 600 anni, e non vi è realtà urbana e rurale europea che non abbia una storia consolidata di presenza di questi gruppi.

La maggior parte dei gruppi ha origini comuni remote, probabilmente in Anatolia, a detta di alcuni nella penisola indiana. Sono comunque arrivati in Europa dopo una lunga permanenza all'interno dei confini dell'impero bizantino. La loro migrazione è sempre avvenuta per piccoli gruppi. Vi sono in tutta Europa, e anche in Lombardia, diversi gruppi chiamati con dispregio "zingari" che hanno traiettorie migratorie assai differenti, e le cui lingue non hanno imprestati significativi dal sanscrito. Si pensi ad esempio ai "dritti", agli "yenish" o ai "caminanti".

La maggior parte di questi gruppi sono sempre stati stanziali, solo alcuni hanno avuto delle abitudini di itineranza per ragioni lavorative. Gli spostamenti di questi gruppi hanno sempre comunque insistito su territori circoscritti, su cui ritornavano ciclicamente.

Forme di migrazione forzata hanno caratterizzato alcuni di questi gruppi, che comunque non si sono mai mossi per grandi numeri, ma sempre sulla base di piccole reti di affinità, non necessariamente di tipo familiare.

La storia dei rapporti fra questi gruppi e la società maggioritaria è assai contestuale, e in nessun modo si può affermare che sia stata una storia di continue persecuzioni. Gli storici hanno mostrato grandi differenze nei rapporti fra gruppi zigani e società maggioritarie a seconda del periodo storico, e anche da città a città. Vi sono diverse tracce storiche, spesso poco conosciute, di ottimi rapporti di complementarietà economica sia in contesti rurali che in contesti urbani. 
Se molti di questi gruppi condividono un dialetto del romanès e chiedono di essere riconosciuti come una minoranza linguistico culturale, nel complesso non per tutti i gruppi in Europa vi è una stessa lingua di riferimento. Anche la religione (cattolica, evangelica, mussulmana, ortodossa) muta da gruppo a gruppo.

Eterogeneità molto forti si riscontrano anche sul piano delle professioni tradizionali. Sebbene molti gruppi (sinti, rudari, dritti) siano stati (e rimangano) coinvolti nell'industria dello spettacolo viaggiante (circensi e giostrai), queste competenze non sono generalizzabili all'insieme dei gruppi. Contadini, operai, artigiani del ferro, mercenari, addetti alla transumanza, allevatori di cavalli, pescatori, etc... l'eterogeneità è il tratto principale da tenere in considerazione.

Nonostante alla fine dell' 800 una certa élite rom (in particolare dell'Est europa) abbia costruito delle rivendicazioni in termini nazionalisti (sulla falsa riga del modello culturale sionista), la maggior parte dei gruppi non ritiene di fare parte di un "popolo". I rapporti di solidarietà interni ai gruppi sono basati su relazioni di affinità scelte, nella maggior parte dei casi più importanti di quelle di sangue e familiari.

I tratti essenziali dell'antropologia di questi gruppi possono essere ricondotti a una forte endogamia matrimoniale e a un certo grado di apertura nella socialità e negli scambi economici. Nell'insieme le culture di questi gruppi sono spesso assai flessibili e adattive.

Come ogni gruppo sociale, anche i rom, sinti, gitani, kale, travellers, yenish, dom, manouches, lom, etc. sono gruppi stratificati. Quando ragioniamo su politiche e interventi a loro rivolti non dobbiamo pensare solo allo strato più povero. Così come è uno stereotipo pensare agli zingari come ladri, ugualmente è stereotipica l'immagine del rom povero che vive nelle baracche. Sono condizioni tragiche e reali, ma che non riguardano l'insieme della popolazione che si auto identifica come rom. A fianco a condizioni di underclass, vi sono in tutti i paesi d'Europa dei ceti popolari, un certo ceto medio soprattutto di commercianti, ma non solo, e ovviamente anche una borghesia rom. Stesso può dirsi per tutti gli altri gruppi.

La continuità di fenomeni di accattonaggio e richiesta di elemosina nelle città lombarde da parte di "zingari" non è da interpretare come una continuità di comportamento da parte di gruppi stabilmente "improduttivi". Le diverse ondate di migrazioni zigane che si sono susseguite hanno spesso portato nelle periferie delle città lombarde gruppi di nuova immigrazione, particolarmente impoveriti, e senza catene migratorie pregresse che hanno avuto comportamenti di questua e anche di furto (alla persona, negli appartamenti). Ma nessun gruppo di cui siamo a conoscenza ha mantenuto nel tempo questi comportamenti (andando sia nella direzione di una forte integrazione, sia - in alcuni casi - nella direzione di atti di maggiore devianza ed illegalità). La presenza di sempre nuovi gruppi zigani nelle città, e la sostanziale misconoscenza della loro artico- 
lazione e eterogeneità, consolidano lo stereotipo di una tradizione culturale immutabile di mendicità e delinquenza.

La loro condizione giuridica è assai eterogenea (cittadini italiani, cittadini di altri Stati membri dell'Unione europea, cittadini di Paesi extracomunitari, rifugiati, apolidi) e non disponiamo di cifre attendibili relativi alle loro proporzioni nelle diverse città lombarde.

Ai fini di una corretta programmazione degli interventi, come sottolineato da Tosi, Cagnoli e Pessina (2007), non è possibile concentrarsi solo su queste prime stime. Da un lato è possibile che in soli quattro anni in molte province le quantità si siano modificate significativamente. Dall'altro, concentrarsi solo sulle persone residenti in campi regolari e irregolari vorrebbe dire disconoscere l'importanza, anche a fini di intervento sociale, di rom e sinti che hanno avuto traiettorie di integrazione di successo e che nella grande maggioranza dei casi nascondono la loro appartenenza (è il caso anche di diversi piccoli imprenditori, per esempio di commerci di carne equina, o di distributori di benzina). Non ultimo, non disponiamo di dati aggiornati sulla presenza - per altro assai significativa - di insediamenti rom e sinti a Novara.

In altri termini, un grande lavoro conoscitivo sulle dimensioni e l'articolazioni dei mondi zigani andrebbe rilanciato. A questo fine le fonti conoscitive istituzionali (polizia locale e assessorati alle politiche sociali) possono fornire solo una informazione parziale. Un lavoro conoscitivo, se non completo comunque maggiormente rappresentativo, richiederebbe una forte collaborazione con i rom e i sinti stessi, finalizzata a ricostruire le reti di legami dentro e fuori gli insediamenti collettivi (campi, micro-aree, terreni agricoli di proprietà, baraccopoli), per avere una stima approssimativa delle persone che vivono con il loro stretto nucleo familiare, pur mantenendo legami con il resto della propria comunità di appartenenza. Questo lavoro più attendibile e più completo richiederebbe un forte rapporto fiduciario con le comunità rom e sinte, dovrebbe garantire il più alto rispetto della privacy e dell'anonimato dei dati individuali e andrebbe progettato in modo da non confondersi in alcun modo con un censimento di carattere etnico.

Stime effettuate in territori differenti da quello lombardo, e perciò assai contestuali e non foriere di indicazioni precise, ci dicono comunque che, seppure con alcune differenze da gruppo a gruppo, il numero di rom non abitanti in campi e insediamenti collettivi, è assai significativo oscillando fra il 50\% e il $200 \%$ di quanti abitano in gruppo. 
Tab. 1 - Insediamenti per tipo e area territoriale

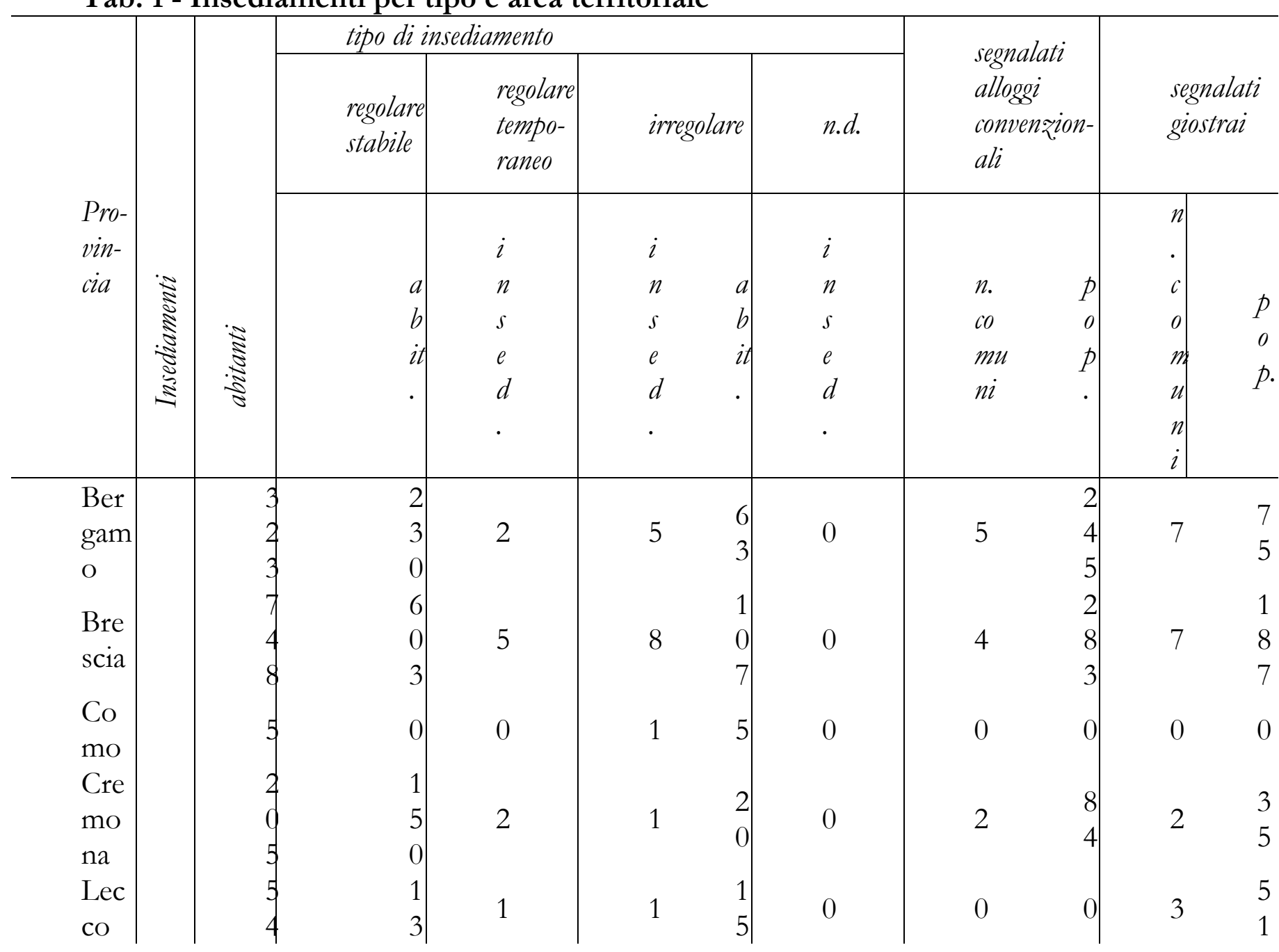




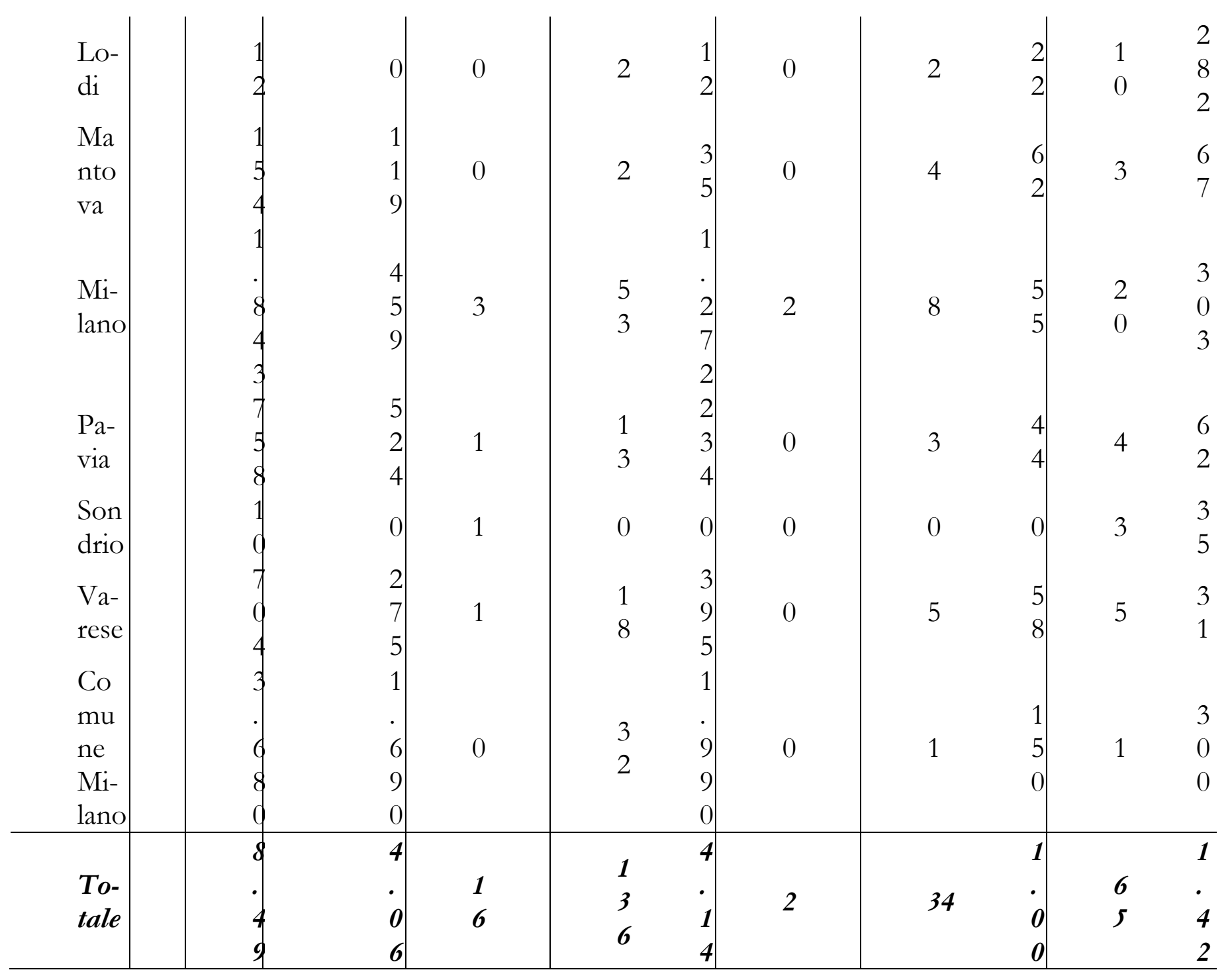




\begin{tabular}{r|r|r|r|r|r|r}
\hline & & $\oint$ & 3 & 8 & 3 & 8 \\
\hline
\end{tabular}

Fonte: Tosi, Cagnoli, Pessina (2007, p. 61). 
Certamente avere a disposizione un quadro più completo della presenza di rom e sinti permetterebbe di distinguere con maggiore precisione non solo $\mathrm{i}$ gruppi, ma anche le traiettorie individuali, e di aggredire così lo stereotipo della miseria, o dell'assistenzialismo. Condizioni che pur riguardando alcune delle persone appartenenti a questa minoranza, non possono essere generalizzate come tratti caratterizzanti e diffusi omogeneamente. Ancora una volta, gli indizi che si possono rintracciare nella letteratura ci fanno parlare di una grande varietà di (1) formule abitative, (2) condizioni lavorative e reddituali, (3) traiettorie migratorie e (4) legami di appartenenza e socialità sulla base di reti di affinità.

I principali problemi

Volendo procedere a una programmazione di interventi e piani di azione di tipo strategico, punto di partenza non esclusivo è l'identificazione di problemi specifici di questi gruppi. Pur cercando di essere parsimoniosi e di accorpare al massimo le mille questioni che si intrecciano nelle esperienze individuali, abbiamo comunque individuato 16 differenti grappoli di problemi, che riteniamo importante elencare mantenendo ben distinti.

La varietà di formule abitative, una sfida per la programmazione

Le formule abitative adottate dai gruppi zigani sono le più disparate. Vi sono persone che vivono sulla base del proprio stretto nucleo familiare in appartamenti di proprietà. Vi sono persone che vivono in appartamenti in affitto. E' banale, ma è bene ricordarlo laddove spesso si pensa che questi gruppi vogliano vivere esclusivamente in roulotte, o in prefabbricati. Non necessariamente quanti vivono in appartamenti sono le persone più giovani, di nuova generazione. In alcuni gruppi sono le persone di mezza età, o rimaste vedove che decidono di andare ad abitare in appartamento, pur mantenendo stretti legami e frequentazioni con gli insediamenti collettivi. In altri casi sono le giovani coppie senza figli. Nuovamente non vi è una regola, o un criterio unico per gruppi così differenti.

La fenomenologia di formule abitative è molto ampia.

Vi sono gruppi che mantengono forme di lavoro itinerante che vivono in roulotte. Non sono "nomadi", perché hanno comunque una città di riferimento, e delle zone abbastanza circoscritte e stabili in cui si recano ciclicamente. Essi richiedono aree di sosta nelle città che toccano ciclicamente e piccole aree di proprietà in cui avere la residenza e tenere come base stabile di radicamento. E' il caso dei caminanti, dei dritti, e di alcuni sinti (a Pavia, a Gallarate, a Saronno, a Busto Arsizio, Mantova per esempio).

Vi sono gruppi che non hanno più spazi di mercato nei mestieri itineranti, ma che vogliono continuare a vivere sulla base dei legami della famiglia allargata (quasi mai sopra le quaranta persone). Non vogliono vivere in campi in cui sono costretti a convivere con persone, anche dello stesso gruppo, ma non della stessa rete familiare (e in alcuni casi, della stessa rete di affinità). Queste perso- 
ne chiedono delle micro-aree da acquistare in cui poter vivere in case senza piani e in cui poter tenere anche la roulotte (importante per spostarsi per le cerimonie religiose collettive, o anche per recarsi con il proprio nucleo familiare a trovare parenti e coltivare le reti di solidarietà lunghe, fondamentali nei gruppi endogamici). E' sempre più il caso di molti dritti e di molti sinti, anche nelle stesse città prima citate (Pavia, Gallarate, Busto Arsizio, Saronno, Mantova, etc), ma anche di molti gruppi di rom harvati e anche di kanharia e di xoraxané. In alcuni casi essi sono in affitto su terreni comunali, o vi risiedono sulla base di contratti di comodato o di enfiteusi. In altri casi, e sono certamente le condizioni migliori, hanno comprato i diritti di proprietà di un terreno ad uso residenziale. In moltissimi casi, invece, hanno acquistato terreni ad uso agricolo per mettervi la casa viaggiante: oggi queste situazioni sono qualificate come abusive, poiché il testo unico dell'edilizia (entrato in vigore il $1^{\circ}$ luglio 2003) ha qualificato come interventi di nuova costruzione anche l'installazione di strutture quali roulottes, campers o case mobili. Per anni associazioni, operatori sociali ed enti locali hanno incoraggiato le famiglie rom e sinte a comprare terreni agricoli a basso prezzo, e lontani dalle periferie delle città, per collocarvi delle case mobili e mantenere uno stile di vita basato sulla famiglia allargata e la condivisione di alcuni spazi aperti. Dal 2003 tutte queste realtà sono qualificate come abusive e vengono sottoposte spesso a sgombero se non a esproprio.

Vi sono gruppi che non hanno mai avuto tradizione di lavoro itinerante, e in cui però le persone vogliono vivere con la propria famiglia allargata. Non richiedono micro-aree in cui poter tenere una casa viaggiante, per loro irrilevante, ma cercano comunque formule abitative ampie, ad esempio cascine. E' il caso di molti rom kosovari, ma anche di alcuni rom rumeni. Vi sono casi interessanti di definizioni di contratti di affitto "associativi" che permettono di regolarizzare al meglio queste forme di abitare collettivo. In molti casi le famiglie rom chiedono di acquisire $\mathrm{i}$ diritti di proprietà delle cascine in questione, con formule ancora da inventare e di difficile equilibrio fra diritti individuali e abitare collettivo.

Vi sono, come abbiamo ricordato all'inizio di questo paragrafo, persone che vivono in appartamenti di proprietà, anche da soli (o con il proprio ristretto nucleo familiare).

Vi sono persone che vivono in appartamenti di edilizia convenzionata. In molte province sono distribuiti in diversi stabili, mentre in alcuni casi (a Milano, ad esempio) sono stati concentrati in pochi edifici, realizzando quelli che a volte sono stati definiti "campi verticali". La loro concentrazione spaziale in singoli edifici di edilizia popolare non risponde necessariamente a una loro richiesta di prossimità, ma più spesso a scelte politiche.

Vi sono persone che vivono in appartamenti in affitto, in cui il contratto d'affitto è garantito dal Comune ai proprietari. Il Comune a sua volta subaffitta alle famiglie rom, sia a prezzo di mercato sia a prezzo calmierato, a seconda della capacità di reddito della famiglia. Questi interventi riducono i costi 
politici di attribuzione di case popolari ai rom, correggono le storture del mercato degli affitti a fronte di un forte stigma nei loro confronti, e abbinano al sostegno abitativo forme di mediazione degli eventuali conflitti di vicinato e forme di patrocinio e assistenza sociale finalizzate ad aiutare i percorsi di inclusione civile, prima ancora che sociale, delle famiglie.

Vi sono persone che vivono in appartamenti di housing sociale aiutati dal terzo settore. In alcuni casi le associazioni affittano sul mercato privato degli appartamenti e li subaffittano ai rom. L'obiettivo è quello di garantire i proprietari e ridurre i problemi di stigma che impediscono l'accesso al mercato dell'affitto.

Vi sono persone che vivono in "campi nomadi" o in "campi comunali", cioè in aree attrezzate dai Comuni, parzialmente regolate in Lombardia con la Lr n. 77 del 22.12.1989 (Azione regionale per la tutela delle popolazioni appartenenti alle "etnie tradizionalmente nomadi o seminomadi") e in Piemonte con la Lr n. 26 del 10.6.1993 (Interventi a favore della popolazione zingara), e in parte da regolamenti preparati dal Prefetto di Milano e dal Prefetto di Torino in qualità di Commissari straordinari all'emergenza nomadi. Non si può formulare un giudizio univoco sull'esperienza assai differenziata dei campi comunali. Molti di essi sono diventati ghetti separati dalla città e dal resto della popolazione, contribuendo all'emergere di quella mentalità del ghetto che tanto danneggia gli abitanti. In questi casi, notiamo come l'isolamento spaziale del campo porti a un isolamento relazionale e finanche professionale delle persone, e un vero e proprio effetto di intrappolamento. Vi sono, però, anche esperienze assai più positive, di campi piccoli con un buon livello di auto-gestione, basati su reti di affinità, in cui convivono persone che vogliono vivere insieme. In queste situazioni emergono spesso anche forme di imprenditorialità basate sul mutalismo cooperativo. Nei casi più positivi, le persone chiedono di poter acquistare i diritti di proprietà se non del terreno almeno delle case in cui risiedono. Esperimenti interessanti sono stati realizzati attraverso l'adozione di contratti di enfiteusi.

Vi sono, infine, persone di recente immigrazione da paesi dell'Est che vivono in insediamenti precari estremamente miseri all'interno di edifici dismessi o di aree abbandonate, e in alcuni casi in boschi o ai margini di canali e torrenti. Sono le cosiddette baraccopoli, che hanno estensioni che comprese fra le 20 e le 300 persone, e che negli anni passati, in alcuni casi, avevano visto insediamenti anche di oltre mille persone (a Milano): oggi tendono ad essere più piccoli e dispersi. Per la fascia più povera e meno dotata di strumenti l'esperienza della baraccopoli può rivelarsi una trappola senza via di uscita, in cui si perdono progressivamente competenze lavorative e autonomia. Per molti, tuttavia, l'abitare in baraccopoli è un comunque un'esperienza temporanea, e vi trovano risorse anche relazionali che, nella prima fase del percorso migratorio, li aiutano a fare il salto verso condizioni abitative più sicure e salubri. Non è ancora chiaro cosa faccia sì che l'esperienza dell'abitare in una baraccopoli sia a termi- 
ne, e foriera di una traiettoria di uscita dall'esclusione, o al contrario tenda a diventare invalidante e perenne. L'aspetto ciclico e ricorsivo degli sgomberi praticati in alcuni comuni tende ad avere più effetti negativi che positivi sulle capacità di autonoma fuoriuscita. Vi sono anche in Lombardia esperienze comunque positive di interventi a bassa soglia per il miglioramento temporaneo della qualità della vita nelle baraccopoli (con forniture di acqua e in alcuni casi di energia elettrica) e di accompagnamento verso altre formule abitative.

In altri termini, la questione abitativa è problematica non solo per le condizioni di indigenza di alcuni individui, ma anche per l'eterogeneità delle richieste formulate dai diversi gruppi. Eterogeneità che richiede buone capacità di programmazione agli enti locali. Ciascuna delle formule abitative esistenti presenta, infatti, specifiche problematiche e richiede adeguate attenzioni.

\section{La condizione giuridica}

Le minoranze rom e sinti hanno quattro caratteristiche specifiche che le differenziano da ogni altra minoranza linguistica presente nel territorio italiano e che implicano complesse esigenze di regolazione giuridica (Bonetti, 2011):

1) gli appartenenti alla minoranza non vivono soltanto in zone circoscritte, ma sono diffusi su tutto il territorio nazionale;

2) appartengono alla stessa minoranza persone che vivono una vita stanziale e persone che vivono una condizione di vita itinerante o semitinerante;

3) tra gli appartenenti alla minoranza è diffuso il desiderio di vivere molto vicini ai componenti della famiglia allargata;

4) all'interno della medesima minoranza si rileva una forte eterogeneità tra i diversi gruppi linguistici e culturali; ognuna delle persone appartenenti alla stessa minoranza ha una condizione giuridica in parte diversa dalle altre in relazione sia al dirittto nazionale che ad altri status garantiti da norme comunitarie o internazionali:

a) vi sono tra $i$ Rom e i Sinti cittadini italiani (circa 6 secoli fa i rom e i sinti si stanziarono in Italia) e secondo molte stime si tratta della maggioranza degli appartenenti alla minoranza.

b) cittadini di altri Stati membri dell'Unione europea (non soltanto Romania o Bulgaria, ma anche Grecia, Spagna, Francia, Germania, Irlanda, Ungheria, Slovacchia);

c) cittadini di Stati extracomunitari;

d) cittadini di Stati extracomunitari a cui è stato anche riconosciuto lo status

di rifugiato o lo status di beneficiario della protezione sussidiaria;

e) apolidi. Molti rom e sinti hanno perduto la cittadinanza dopo l'estinzione

di alcuni stati plurinazionali, come l'ex Jugoslavia, senza acquistare la cittadinanza di altro Stato, oppure hanno dovuto lasciare il territorio del loro 
Stato a causa di persecuzioni per motivi etnici o di conflitti interni o internazionali.

Molte raccomandazioni del Consiglio d'Europa, dell'OSCE e degli organismi dell'Unione europea mirano a dare una specifica attuazione alle tutele minoritarie nei confronti dei rom e dei sinti. La raccomandazione 1203 (1993) del 2 febbraio 1993 del Consiglio d'Europa afferma che rom e sinti necessitano di una protezione particolare e ritiene che le disposizioni di ogni protocollo addizionale o convenzionale relativo alle minoranze dovrebbero applicarsi alle minoranze sprovviste di territorio. Tuttavia la presenza consistente in Italia di non italiani tra i rom e i sinti che si trovano nel territorio dello stato complica la disciplina giuridica: le difficoltà derivano non tanto dalla disciplina del trattamento, ma anzitutto dalla disciplina dell'ammissione e della permanenza nel territorio dello stato degli appartenenti alla minoranza, qualora si tratti di non cittadini.

\section{I conflitti locali con la società maggioritaria}

La diffusa ostilità nei confronti di rom e sinti e l'avversione per la loro presenza entro determinati territori ha dato vita a diverse mobilitazioni locali. Nonostante la diffusione di sentimenti antizigani abbia una dimensione nazionale, la mobilitazione ha carattere locale perchè le dinamiche del «panico morale» si originano a partire da fenomeni, sentiti come pericolosi, relativi a gruppi specifici e situati, visibili e connessi alla vita quotidiana. Difficilmente le mobilitazioni di cittadini che si oppongono alla presenza stabile di rom e sinti sul proprio territorio hanno un carattere «spontaneo». Sono invece il risultato dell'azione di imprenditori politici (partiti della destra e dell'estrema destra), di azioni collettive in cui gli attori sollevano problemi locali rendendoli pubblici e interagendo con le autorità, in vista in vista del raggiungimento di determinati obiettivi. Tra questi obiettivi gioca un ruolo centrale il conseguimento del consenso politico.

Il territorio e la dimensione locale sono la 'base materiale' di queste azioni collettive: la costruzione dell'attore conflittuale (spesso comitati di cittadini) origina dall'esistenza di un confine perimetrato da attori esterni, che minacciando la 'comunità' contribuiscono a crearla. Vengono denunciate l'arroganza del potere e l'esautorazione dei cittadini da un decisione che riguarda direttamente le loro vite, come quella di realizzare un insediamento rom. Si interpreta la propria azione come una reazione contro attori sociali la cui condizione è connotata dal privilegio: il privilegio degli "zingari", a cui viene imputato di essere assistiti in modo privilegiato dalle istituzioni pubbliche, privilegio a cui la 'gente comune' autoctona non può avere accesso.

La paura, l'emergenza, la sicurezza, la percezione dell'assenza delle istituzioni nel governo dei problemi del territorio, della loro mancanza di imparzialità rispetto ai conflitti, sono meccanismi di attivazione della protesta. La protesta ha 
per bersaglio persone e strutture che vengono identificate con il 'degrado sociale', che vengono attaccate proprio in quanto rappresentano un rischio di contagio con il disagio, e vive di un processo di polarizzazione dovuto in primo luogo all'assenza di istanze di mediazione istituzionale. I conflitti tra società maggioritaria e insediamenti rom e sinti poggiano sull'assenza di contatti e interazione tra i due ambiti. Ma la loro premessa fondamentale è l'altissimo tasso di diffidenza nei confronti di queste popolazioni.

\section{Il razzismo e la discriminazione}

Da un'indagine campionaria realizzata da Eurostat nel 2008 (Commissione europea, 2008), basata sull'interrogativo "Come ti sentiresti a sapere che il tuo vicino è un Rom?", emerge che solo il 14\% del campione italiano si sentirebbe pienamente a proprio agio, e solo il 5\% dichiara di avere rapporti personali con almeno un rom o un sinto. Il pregiudizio nei confronti di queste minoranze è diffuso in tutta Europa, ed è ovunque maggiore rispetto a quello che riguarda le altre minoranze. Dalla stessa indagine Eurostat si evince che tra i 27 paesi dell'Ue la percentuale di chi si sentirebbe «comfortable» a vivere a stretto contatto con rom e sinti è solo del 36\%, mentre solo il 14\% ha rapporti personali diretti con loro. Quest'ultimo dato indica un bassissimo livello di interazione tra società maggioritaria e rom e sinti, con la parziale eccezione dei paesi dell'est europeo e della Spagna. Questo dato a sua volta contribuisce alla percezione distorta della cultura di rom e sinti. A tale percezione contribuiscono notevolmente anche i media, la cui rappresentazione di rom e siniti è spesso basata su immagini superficiali e stereotipiche, che li descrivono come artisti o criminali, associandoli a immagini di degrado urbano e a problematiche sociali quasi sempre riconducibili a temi dell'ordine, del decoro e della sicurezza.

La percezione di rom e sinti è quindi pregiudizialmente negativa in tutta Europa. Ma con differenze. E le differenze fra i diversi Paesi permettono di denaturalizzare i pregiudizi. In Italia la percezione è assai negativa: il $62,8 \%$ della popolazione dichiara di non volere rom o sinti come vicini di casa. Tra il 1999 e il 2008 tale percentuale è cresciuta di circa il 7\%. Rimane quasi invariata la percentuale di coloro che pur definendosi tolleranti verso le differenze etniche mantiene elevati livelli di ostilità anti-zigana. Solo il 6,7\% degli italiani dichiara di non avere ostilità verso questi gruppi (Arrigoni, Vitale, 2008). Al contrario di ciò che avviene per i pregiudizi verso altre etnie, i sentimenti anti-zigani non decrescono al crescere del titolo di studio e sono quasi del tutto indifferenti alla frattura politica destra/sinistra. Lo "zingaro" è in Italia associato all'immagine del ladro per il 92\% della popolazione (indagine Ispo, 2007), vive in un gruppo chiuso $(87 \%)$, risiede per propria scelta in campi ai margini della città $(83 \%)$, e in molti casi sfrutta i bambini $(92 \%)$. La maggior parte della popolazione colpevolizza interamente rom e sinti per le situazioni di deprivazione che vivono: il 57\% degli italiani pensa che il miglioramento delle loro condi- 
zioni di vita dipenda dal rispetto di leggi e regole da parte loro, il $32 \%$ dall'uscita da un atteggiamento questuante e para-assistenzialistico. Solo una minoranza pensa che a questo fine siano necessarie specifiche politiche sociali.

Grafico n. 1. Percentuale di persone che dichiarano di non volere Rom o Sinti come vicini di casa.

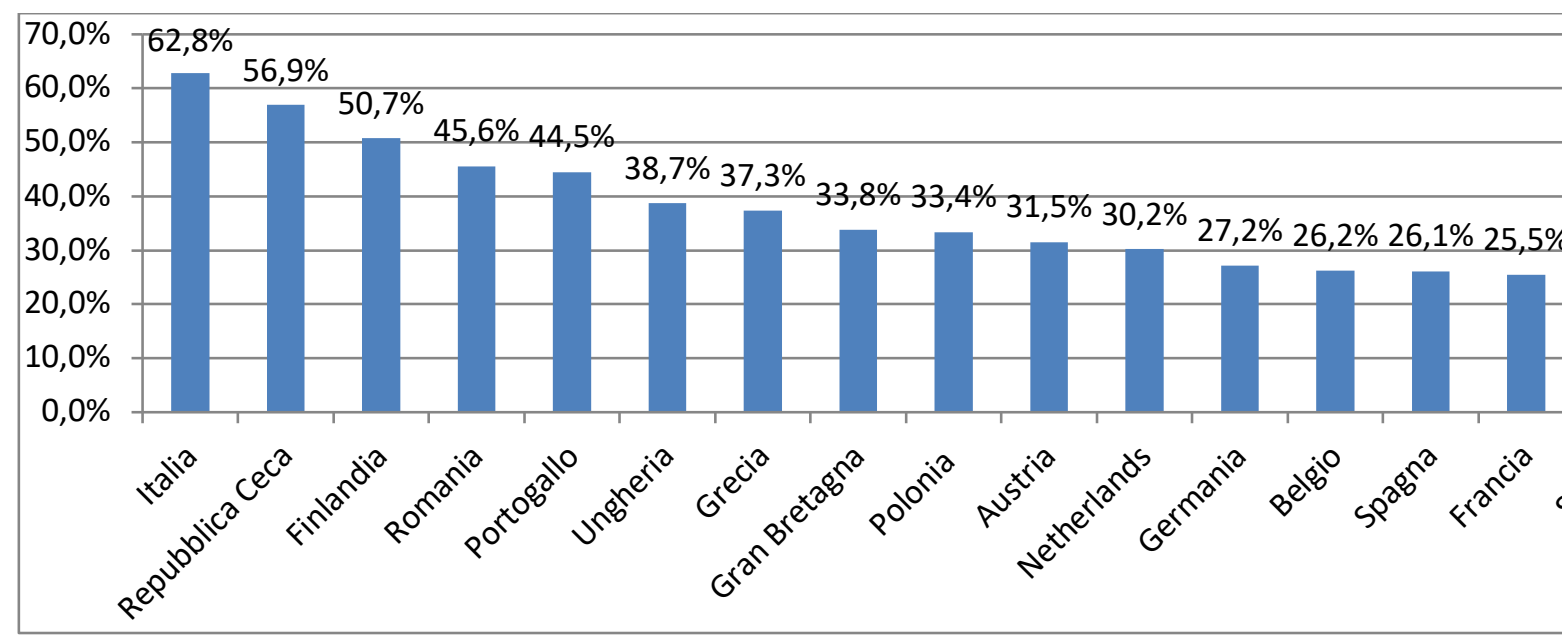

Fonte: European Value Survey, 2008. Elaborazione: Vitale, Claps, Arrigoni, 2011.

Così come una generica ostilità non si traduce automaticamente in mobilitazione conflittuale, ma necessita di mediazioni politiche per trasformarsi in protesta, così il pregiudizio culturale non ha una dimensione naturale. È invece attivamente costruita da attori sociali all'interno di un campo di discussione, di conflitto e di interazione pubblica. Tra pregiudizio antizigano e politiche discriminatorie vige un rapporto di reciprocità che potremmo definire «spirale del consenso». La situazione di rom e sinti in Italia è affrontata dagli attori della politica ufficiale prevalentemente attraverso lo schema campi-ciclo degli sgomberi (Vitale 2010). Poichè le popolazioni zigane sono quelle percepite con minor simpatia della popolazione e la loro presenza è comunemente associata a problemi di degrado e sicurezza, gli attori politici ritengono che l'intervento più remunerativo sul piano del consenso sia quello di agire attraverso cicliche prove di forza, che diano l'immagine di una politica efficiente nella gestione del «disordine». Una politica pubblica incentrata sulla reiterazione dello sgombero dei campi - dopo che in Italia, caso unico in Europa, la stessa istituzione dei campi è stata per diverso tempo l'unico intervento di politica pubblica rivolto a queste popolazioni - implica a sua volta il rafforzamento delle rappresentazioni mediatiche e delle percezioni pubbliche che associano minoranze 
zigane e problemi ordine pubblico, e un'intensificazione della definizione di queste popolazioni attraverso sequenze logiche puramente sottrattive, definite cioè in base a mancanze ed assenze rispetto alla rappresentazione standard del cittadino virtuoso: scarsa propensione alla partecipazione scolastica e lavorativa, scarsa volontà di ricercare sistemazioni abitative alternative al campo, incapacità di rispettare regole e leggi. Tali processi retroagiscono ulteriormente sui livelli di ostilità diffusa da parte della società maggioritaria, accrescendoli e creando le basi per nuove misure incentrate su dispositivi securitari. La spirale del consenso basata sul ciclo dello sgombero è da intendersi come politica demagogica, cioè come politica che ricerca il consenso agitando un tema su cui non vi è l'attenzione spontanea dell'opinione pubblica, astraendo dalle condizioni e dai processi che determinano una o più situazioni sociali così come dagli effetti concreti delle politiche adottate. L'adozione di un'agenda pubblica rivolta a rom e sinti basata sul ciclo "spirale del consenso"-sgomberi, non produce risultati reali sui problemi che vengono evocati per legittimare tali interventi e implica un riduzionismo che conduce le istituzioni ad abbandonare altri tipi di intervento politico, come le politiche di assistenza sociale, le politiche attive del lavoro, i servizi di mediazione culturale e di accompagnamento scolastico. A rom e sinti viene così riservato un trattamento discriminatorio che tende a ridurli a scarti, inferiorizzati, spazialmente segregati, stereotipizzati.

\subsection{Criminalizzazione e criminalità}

La diffusione di immagini stereotipizzate dei Rom come criminali segue meccanismi assai noti nello studio delle dinamiche dell'opinione pubblica. Il comportamento di singoli viene indebitamente generalizzato all'insieme di una popolazione ritenuta avere alcune caratteristiche in comune. La base di questo meccanismo risiede nella mancanza di conoscenza di casi diversi da quelli noti nello spazio pubblico perché legati a criminalità, comportamento deviante e infrazione della legge. Esattamente come nel caso dell'immagine della miseria e della vita nelle baraccopoli, il fatto che non si conosca l'insieme complesso di condizioni di vita di queste persone, porta a stereotipi pesanti e gravidi di conseguenze.

Questa attenzione ai meccanismi del razzismo nelle dinamiche dell'opinione pubblica non deve tuttavia farci chiudere gli occhi di fronte agli episodi più o meno gravi di illegalità compiuti anche dagli appartenenti a questa minoranza. Le ricerche mettono in luce due punti principali su cui vale la pena insistere selettivamente in vista della progettazione di interventi di promozione sociale da realizzare.

Il primo punto concerne gli aspetti di micro-criminalità, e in particolare il furto e il borseggio. Nonostante alcune balzane teorie pseudo-antropologiche, mai validate dalla comunità scientifica, secondo cui vi sarebbe una certa propensione culturale al furto da parte degli "zingarelli" e delle "zingarelle" perché in- 
scritti in comunità di "raccoglitori" o per disprezzo verso la proprietà di chi non riconoscono come Rom, nessun riscontro empirico è risultato comprovare queste ipotesi culturaliste. Semmai quello che le indagini hanno ben messo in luce è che, essendo questi gruppi assai diversi e provenienti da diversi Paesi, senza solidarietà fra loro, all'inizio di un processo migratorio, in assenza di catene migratorie familiari pregresse, alcuni di questi gruppi hanno avuto la tendenza a vivere di espedienti e ad adottare comportamenti di micro-criminalità. Raramente questi comportamenti hanno avuto una durata superiore ai quindici anni. In altri termini, dopo un certo periodo di tempo, i gruppi trovano altre modalità di inserzione nelle società locali, e abbandonano i comportamenti devianti. Le indagini più serie hanno documentato come non vi sia quindi continuità. Il fatto che vi siano degli "zingari" che borseggiano oggi come vent'anni fa o quarant'anni fa, non vuol dire che vi sia un gruppo deviante che perpetua comportamenti criminosi e anti sociali nel tempo. Chi borseggiava negli anni '60 (soprattutto Xoraxané), o nella seconda metà degli anni '80 (soprattutto Kanharija) non è più dedito a queste attività. Ugualmente sono state abbandonate le pratiche di richiesta di elemosina. La presenza di una nuova immigrazione rom dalla Romania con alcuni comportamenti similari negli ultimi 10 anni ha fatto pensare a molti a una continuità di pratiche da parte di un gruppo etnico omogeneo, in cui la devianza si riproduce immutabile nel tempo. Non è così: parliamo di gruppi diversi, in assenza di continuità. La spiegazione del fenomeno è da attribuirsi, semmai, all'assenza di catene di solidarietà all'inizio delle nuove ondate migratorie. Assenza che lascia le persone in forti difficoltà rispetto alla ricerca di reddito, prestigio e domicilio.

Un secondo punto su cui ha insistito la ricerca (non in Italia, a dire il vero, ma in altri Paesi europei) attiene invece alle traiettorie individuali di affiliazione alla criminalità organizzata. Parliamo precisamente di traffico d'armi, di automobili, e di attività di vendita senza pagamento di tasse di produzioni industriali non tracciate (soprattutto elettrodomestici), e in alcuni casi di traffico di persone e induzione alla prostituzione. La letteratura mostra come condizioni di segregazione spaziale e sociale elevate (sia in campi nomadi, sia nei cosiddetti campi verticali, edifici di edilizia popolare altamente segregati sul piano etnico e sociale) rendono più vulnerabili i gruppi alle proposte della criminalità organizzata. Non sembra riscontrabile alcuna specificità dei gruppi rom e sinti rispetto ai noti meccanismi e processi di coinvolgimento nella criminalità organizzata. Con riferimento specifico alle provincie lombarde, senza poter disporre di ricerche appropriate, a nostra conoscenza ed esperienza, anche per collaborazione e insegnamento con le forze di polizia locale, possiamo ipotizzare che il problema si ponga in maniera più intensa a Milano e nella sua provincia, e in particolare nei quartieri tradizionalmente molto condizionati dalla criminalità organizzata. Qui i rom, in particolare laddove vivano in campi, tendono ad essere reclutati dalla criminalità organizzata. Il punto su cui vogliamo insistere è che a nostra conoscenza, così come nella letteratura su altri Paesi, non risulta 
che i gruppi rom diano vita a proprie forme organizzate di criminalità, ma semmai siano, in alcuni casi, reclutati come manovalanza dalle organizzazioni criminali esistenti. La questione è densa di conseguenze in termini di politiche e interventi possibili, perché indica non vi sia una specificità, ma l'esigenza di procedere a politiche di de-segregazione, e a fornire alternative alle proposte di lavoro della criminalità, in particolare nelle situazioni in cui la criminalità organizzata è l'unico soggetto ad offrire opportunità di reddito.

\section{La debole rappresentanza e la frammentazione degli interventi}

Due ulteriori elementi determinano la centralità di strumenti quali lo sgombero o la segregazione estrema nei "campi nomadi" e, parimenti, la diffusione di sentimenti di ostilià nei loro confronti. Il primo è quello della rappresentanza di rom e sinti. È consustanziale sia a politiche incentrate sul ciclo degli sgomberi che a fenomeni di conflittualità legati alla loro presenza l'assenza di una interlocuzione diretta con leader e dirigenti delle comunità. Essi non vengono coinvolti attivamente nei processi di discussione e decisione e non sono considerati attori legittimati a partecipare alle arene pubbliche che si costituiscono per affrontare i problemi connessi alla loro condizione. La rappresentanza non è un portato culturale spontaneo dei gruppi sociali, è una costruzione sociale legata a condizioni istituzionali che la promuovono offrendo una cornice in cui possa formarsi e svilupparsi. Nei contesti caratterizzati da rapporti molto conflittuali con le minoranze rom e sinte (soprattutto, in Italia, le grandi città, in particolare Milano e Roma), le istituzioni elettive, prefettizie e di polizia, tendono a negare loro una capacità di azione diretta nello spazio pubblico. $\mathrm{Al}$ contrario, come emerge da diverse indagini (Parlamento europeo 2008, Coses 2010), le politiche efficaci implicano sempre un coinvolgimento diretto dei destinatari e un riconoscimento della loro capacità di azione.

Il secondo elemento è la presenza di specifici dispositivi istituzionali in cui la rappresentanza di rom e sinti possa essere riconosciuta e contribuire alla discussione e alla decisione pubblica. I dispositivi sono contesti istituzionalizzati e continui nel tempo di interazione tra gli attori e tra le parti in conflitto, dotati di regole e procedure codificate, che consentono la costruzione di un sistema di relazioni tra minoranze rom e sinti, istituzioni, attori politici, attori della società civile e del Terzo settore che svolgono opera di intervento e mediazione con le comunità.

In assenza di dispositivi legittimi la relazione tra attori è caratterizzata da una asimmetria di potere tale che questi gruppi non riescono a costruire azione politica, rimanendo legati a logiche di sussistenza e invisibilità. La presenza di contesti istituzionalizzati di questo tipo è importante per il coordinamento delle stesse istituzioni tra loro, ai fini della costruzione di politiche pubbliche integrate e basata sulla verifica dei risultati. Tipica delle politiche più inefficaci è infatti la frammentazione degli interventi rivolti a rom e sinti. In diversi contesti 
si producono provvedimenti basati esclusivamente sulla logica del controllo sociale e della repressione, come abbiamo visto, o alternativamente incentrati sul problema abitativo, su quello dell'inserimento scolastico o sull'attivazione lavorativa. Da un punto di vista procedurale, gli enti locali tendono a concentrare l'implementazione dell'azione pubblica su una singola struttura (per esempio su un singolo assessorato), invece che predisporre uno stabile coordinamento tra diversi assessorati e tra questi e gli uffici amministrativi preposti. In secondo luogo, come già accennato, la frammentazione si produce tra istituzioni diverse: le istituzioni politiche e quelle prefettizie e di polizia tendono ad agire in relativa autonomia, senza che si possa escludere tra loro un'inversione dei ruoli, con le prefetture impegnate ad individuare soluzioni politiche ai conflitti e alle emergenze e gli enti locali impegnati nella raccolta del consenso attraverso politiche differenzialistiche.

\section{La scolarizzazione}

In Italia solo il $30 \%$ dei minori rom e siniti risulta iscritto alla scuola dell'obbligo, ma la frequenza effettiva è minore rispetto a questa stessa cifra. I problemi che riguardano il rapporto tra comunità rom e sinti e scolarizzazione sono di due ordini diversi, strettamente interrrelati tra loro (Sacco, 2008). Il primo ordine di fattori riguarda la percezione della scuola da parte di rom e sinti. La partecipazione scolastica raramente è percepita, innanzitutto dagli adulti e dai genitori, come utile ad un ingresso nel mondo del lavoro e all'integrazione nella società circostante. Si pensa che essa possa costituire uno sforzo inutile sia per i minori che per le loro famiglie, anche per il fatto che i «tempi di vita» di comunità rom e sinte e società circostante sono diversi: tra le prime, i minori in età da scuola media sono ritenuti già adulti. In secondo luogo vi è il timore, fondato, che i minori rom e sinti che partecipano alla vita scolastica debbano andare incontro a processi di isolamento e stigmatizzazione e a diverse difficoltà di adattamento nei rapporti con gli altri minori e gli insegnanti. In terzo luogo vi sono atteggiamenti di difesa della propria cultura e identità rispetto alla cultura dominante rappresentata dalla scuola e dal modello educativo in essa vigente. Una parte delle famiglie rom e sinte ritiene che la partecipazione scolastica dei propri figli possa comportare una perdita di potestà educativa da parte loro e un progressivo distanziamento dei minori dai valori, dagli impegni e dai progetti che definiscono l'assunzione del proprio ruolo nella comunità. Le regole e i codici vigenti nel mondo scolastico possono essere inoltre percepiti come estranei e poco comprensibili, relativamente al modo in cui la giornata scolastica è scandita e organizzata, alle regole su assenze e presenze, allo scambio tra scuola e famiglia e all'accesso ai servizi di mensa e ad altre facilitazioni allo studio. Vi sono infine difficoltà legate alla lingua e al passaggio da una cultura prevalentemente orale a una cultura scritta. 
Il secondo ordine di fattori riguarda il modo in cui l'istituzione scolastica affronta l'insieme di criticità rilevate finora. La più grande carenza del mondo scolastico nei confronti di rom e sinti è stata finora, in Italia, la scarsa comprensione delle problematiche specifiche di queste minoranze. I casi di successo nell'insermento scolastico di rom e sinti sono casi di conciliazione tra le due polarità della «diferenza culturale» e dell'universalismo dell'educazione scolastica. La scuola, istituzione a vocazione necessariamente universalistica e in una certa misura omogeneizzante, è riuscita ad integrare queste minoranze laddove ne ha compreso e accolto la specificità. I problemi e gli insuccessi si verificano invece quando, come sempre avviene per fenomeni sociali costituiti da polarità reciprocamente correlate, si ipostatizza uno dei due poli: il polo della differenza ("sono troppo diversi per integrarsi") o il polo dell'universalismo e dell'omogeneità delle forme e dei contenuti dell'insegnamento ("se vogliono inserirsi nel mondo scolastico devono rinunciare alla propria differenza"). Nel primo caso si ottengono politiche differenzialiste. Nel secondo politiche di tipo assimilativo, spesso accompagnate da un forte investimento in interventi basati sul controllo, che prevedono sanzioni gravi in caso di mancato rispetto dell'obbligo scolastico (Marinaro, 2003). L'approccio differenzialista fallisce perchè lascia inalterato il rapporto tra gruppi zigani e scuola. L'approccio assimilativo perchè rafforza la diffidenza delle comunità zigane per una istituzione percepita come estranea e poco funzionale alle proprie aspettative di vita.

La ricerca comparativa italiana sull'inserimento dei minori sinti e rom a scuola (2000-2003) mostra come laddove a scuola i bambini provenienti da un gruppo zigano non sono oggetto di misure educative specifiche, l'efficacia della scolarizzazione è maggiore (Piasere, 2007). Alfredo Alietti (2009), inoltre, dopo anni di indagine etnografica con i sinti di Voghera, ha proposto un quadro analitico estremamente raffinato e stimolante per ragionare sui processi di scolarizzazione e sulle modalità di intervento educativo extrascolastico dentro un quadro di duplice tensione, con il contesto esterno ma anche intergenerazionale all'interno dei gruppi. Ne ricava indicazioni a non contrapporre il minore sinto alla propria famiglia e a pensare percorsi di sostegno ed emancipazione mai solo per i bambini ma, differenziati, anche per gli adulti: se si vuole sostenere la scolarizzazione dei bambini occorre mostrare il vantaggio della formazione anche agli adulti.

Un ruolo centrale nel fallimento delle politiche scolastiche per rom e sinti lo gioca la segregazione spaziale. La loro collocazione in spazi periferici, isolati e non integrati nella vità della comunità locale non facilita lo scambio e il reciproco avvicinamento tra comunità e scuola. Inoltre, essendo strettamente connesso alle condizioni abitative, al rapporto con la società maggioritaria e alla rappresentazione della funzionalità dell'educazione ai fini della vita lavorativa, il problema dell'inserimento scolastico non può essere affrontato separatamente dalle politiche abitative e dalle politiche di inserimento lavorativo. 
Infine, centrali in tutti $\mathrm{i}$ casi di politiche efficaci in questo campo sono la mediazione linguistica e culturale tra comunità zigane e mondo scolastico, da condurre sia nelle comunità che durante l'orario di frequenza scolastica, che l'organizzazione di attività di scambio e di reciproca conoscenza tra i due contesti, che socializzino progressivamente l'istituzone universalistica della scuola alla «diferenza zigana» e questa all'universalismo educativo.

Non è perciò romanticismo o "buonismo" ricordare come in molte città italiane ci siano ad esempio bambini rom, e non sono pochi, che vanno a fare volontariato nelle residenze sanitarie assistite per tenere compagnia agli anziani (non rom), grazie al supporto di gruppi di volontariato. I gruppi informali che si costituiscono a supporto delle comunità rom sono assai importanti non solo per aiutare i rom nell'accesso alle opportunità scolastiche, ma anche per "inventare" esperienze di formazione professionale, che coinvolgono non solo i più giovani, ma l'intero gruppo familiare.

Le esperienze "informali" di formazione professionale, che individuano delle piccole nicchie produttive e favoriscono la formazione e l'inserimento professionale coinvolgendo piccole imprese familiari, hanno un alto tasso di effettività. Perché? Tre ragioni: (1) si rivolgono all'intero gruppo familiare, e non a singoli giovani, e quindi si fanno forza sui legami familiari, invece di metterli in tensione; (2) sono in grado di coinvolgere piccole e medie imprese radicate territorialmente, producendo una formazione altamente specifica e professionalizzante (con i limiti dell'assenza di certificazione); (3) sono costruite nell'interlocuzione e nella discussione diretta con i rom stessi, e non a tavolino a partire da rappresentazioni stereotipate della cultura rom e del suo presunto degrado.

\section{Avvertenze di metodo nella costruzione di politiche per i gruppi ziga- ni}

La situazione dei gruppi zigani è quella di una forte esclusione sociale complessiva. Esclusione dall'accesso al lavoro, all'educazione, alla sanità e ai servizi sociali. Sono particolarmente diffusi tra questi gruppi - in misura maggiore rispetto a tutte le altre minoranze - analfabetsimo e segregazione spaziale, isolamento e mancanza di servizi essenziali. Essi ricorrono raramente all'assistenza sanitaria pubblica, sia a causa di attitudini discriminanti da parte di strutture e personale sanitario e della segregazione spaziale in cui vivono, sia per la presenza di una parziale conflittualità tra le forme del trattamento sanitario e le attitudini più diffuse di cura della persona e concezione del pudore tra $i$ gruppi zigani, rafforzate dall'assenza di comunicazione tra comunità zigane e servizi sanitari pubblici e dall'assenza di informazioni reciproche. Si tratta, come nel caso della scuola, del conflitto e della reciproca estraneità tra una differenza culturale e un sistema universalistico, tra i quali la politica e le istituzioni sono chiamate a creare forme di mediazione e conciliazione. 
La scarsità di formazione e qualificazione professionale, unitamente a capacità professionali tradizionali sempre più scarsamente richieste dal mercato del lavoro, oltre che, ancora una volta, all'isolamento spaziale nel quale i gruppi più marginali vivono, costituiscono barriere all'ingresso di rom e sinti nel mercato del lavoro. Difficoltà provengono anche dal complesso status legale di questi gruppi e dalle discriminizioni, dirette o indirette, basate sul pregiudizio di una loro "naturale" scarsa inclinazione al lavoro e al rispetto delle regole.

L'esclusione sociale che colpisce rom e sinti si presenta dunque come un fenomeno dovuto a diversi fattori causali interconnessi, che si combinano tra loro in una causazione cumulativa all'interno di un processo multidimensionale e intergenerazionale. La segregazione spaziale, per esempio, ha un impatto diretto sulla salute e sull'accesso ai servizi, così come la scarsa scolarizzazione ha effetti negativi sulle capacità di attivarsi nel mondo del lavoro, ciò che a sua volta rende più difficile la costruzione di percorsi di uscita dalla segregazione spaziale nei campi. Ogni fattore è connesso agli altri ed ha sugli altri effetti retroattivi e rafforzativi. Se si può individuare un fattore che però è al centro di tutte le relazioni causali questo è senz'altro quello della segregazione spaziale, centrale nel co-determinare tutte le altre forme di esclusione e deprivazione.

Date queste caratteristiche dei fenomeni di esclusione sociale e deprivazione di rom e sinti, le ricerche condotte a livello italiano (Vitale, 2008) ed europeo (si veda più avanti il paragrafo 9.12), conducono ed enucleare un preciso set di caratteristiche di politiche pubbliche che abbiano ragionevoli possibilità di avvicinare gli obiettivi della riduzione dell'esclusione e dell'ampliamento dell'integrazione:

a) Varietà degli strumenti di politica pubblica e integrazione tra essi e tra le strutture e gli attori che implementano le politiche

b) Costruzione di processi e meccanismi stabilizzati e continui nel tempo, ma modificabili in base all'esperienza e ai risultati, di traduzione tra comunità zigane e società maggioritaria. Per traduzione si intende in questo caso conoscenza reciproca della cultura, dei codici e dei processi che guidano i rispettivi atteggiamenti e comportamenti sociali.

c) Costruzione e stabilizzazione di dispositivi istituzionali che costruiscano e regolino ambiti di scambio, discussione, presa di decisione e coordinamento delle politiche tra le diverse istituzioni e tra istituzioni, comunità zigane e attori della società civile.

d) Garantire alle politiche continuità nel tempo e verificabilità dei risultati in funzione del loro progressivo adattamento agli obiettivi da raggiungere.

e) Protagonismo diretto degli attori politici degli enti locali nella costruzione e realizzazione delle politiche e nella mediazione dei casi conflittuali

d) Intervenire attivamente nella decostruzione e rimozione dei due principali fattori strrutturali alla base dell'esclusione di rom e sinti: segregazione spaziale e diffusione del pregiudizio. 
Vediamo più nel dettaglio questi diversi aspetti.

A partire dagli anni Ottanta il modello prevalente di politiche per rom e sinti è stato quello incentrato sul binomio campo "nomadi"-sgombero. Le altre forme di intervento sono state o annullate in funzione di questo modello, o ricondotte ad esso, e quindi in gran parte vanificate. Questo però non è l'unico modello di intervento possibile e praticato. Anche in Italia stanno emergendo forme di moltiplicazione degli strumenti di intervento da parte dei governi locali. Una modificazione negli strumenti messi in opera dagli enti locali è importante anche perchè ha implicazioni costitutive sugli attori implicati, poichè le capacità degli attori sociali dipendono fortemente dagli strumenti di politica pubblica impiegati e dagli spazi di azione che essi aprono.

L'univocità della sistemazione in campi "nomadi" può essere così superata da una serie eterogena di soluzioni abitative: abitazioni di produzione pubblica; abitazioni di produzione privata, con attività di sostegno ai membri delle comunità per quanto riguarda l'accesso al credito e la reputazione delle famiglie (ai fini di affrontare e superare il pregiudizio che la coabitazione con famiglie rom e sinti spesso comporta); autocostruzioni su terreni di proprietà delle famiglie, con il sostegno di cooperative; predisposizione di aree attrezzate in funzione residenziale per gruppi familiari estesi, che li gestiscano in proprietà o in affitto; interventi di edilizia pubblica rivolti, attraverso bandi pubblici, anche ma non solo a famiglie zigane, per non incentivare il pregiudizio sui "privilegi" dei rom e non indugiare in schemi differenzialistici; aree di sosta attrezzate per i gruppi itineranti (per esempio i giostrai).

La pluralità degli interventi non riguarda solo le politiche abitative. Per quanto riguarda la scolarizzazione, sono stati sperimentati percorsi che prevedono: la promozione della cultura rom all'interno delle classi; il sostegno educativo (riguardante la partecipazione ad attività collettive, come attività sportive e laboratori creativi) e didattico (direttamente legato all'apprendimento e al successo scolastico) sia all'interno delle classi (il secondo) che, nel pomeriggio, all'interno delle stesse comunità; programmi indirizzati non solo ai minori, ma alle famiglie nel loro insieme, al fine di agire sugli ostacoli culturali presenti tra gli adulti rom e sinti circa la partecipazione dei minori e delle donne alle attività scolastiche; gite culturali con i minori rom e sinti affichè conoscessero, $\mathrm{e}$ in qualche misura iniziassero a sentire propri, i luoghi che circondano gli spazi da loro abitati; sistemi di ricompensa ("premi") per i minori, legati al loro successo scolastico; consultazione delle comunità nel loro complesso prima di avviare i progetti di inserimento scolastico; realizzazione di spettacoli artistici, teatrali, eventi culturali per avvicinare mondo della scuola e comunità rom e sinte; presenza di mediatori specificamente, e approfonditamente, formati allo scopo specifico di intervenire nel rapporto minori-comunità-scuola. Come negli altri ambiti di intervento, è importante che i progetti seguano percorsi sia individualizzati che orientati ai singoli gruppi familiari. 
Per quanto riguarda il lavoro, si sono sperimentate politiche orientate all'accrescimento delle opportunità e (quindi) delle capacità dei singoli e delle famiglie, allo sviluppo del capitale sociale e delle comunità, valorizzando le risorse, le reti e le capacità di autorganizzazione di rom e sinti, secondo un modello di «active welfare approach» che tiene conto sia dei fattori diretti (pregiudizio, isolamento, scarsa formazione e dotazione professionale), che indiretti (abitazione, scuola, accesso ai servizi sociali, legami con la società maggioritaria) di esclusione dal lavoro. Una parte importante dei progetti di inserimento lavorativo prevede una serie di attività propedeutiche: comprensione, da parte di rom e sinti, del contesto urbano complessivo in cui si trovano ad abitare e operare; sviluppo delle abilità ad interagire con la società circostante e le istituzioni; sviluppo della capacità di comprensione e di interazione con il mercato del lavoro.

Ai fini dell'inserimento attivo in realtà lavorative si sono sperimentate convenzioni con aziende e cooperative per corsi di formazione e forme di mediazione di attori pubblici e del terzo settore tra rom e sinti e aziende. Uno dei punti deboli dei progetti di inserimento lavorativo è proprio la difficoltà ad incontrare la disponibilità di attori privati, nonostante la mediazione di enti locali e associazioni, ad offrire opportunità lavorative. Tuttavia, come nel caso delle politiche abitative, la mediazione è una condizione necessaria, anche se non sufficiente, per conseguire i livelli di capitale reputazionale e affidabilità necessari ad erodere presso i privati i pregiudizi nei confronti della capacità lavorativa di rom e sinti. In questi casi si procede alla costruzione di reti tra istituzioni pubbliche, attori del terzo settore, privati, rom e sinti, che necessitano, per dare risultati, di finanziamenti stabili e continuità organizzativa. Attraverso, in particolare, il supporto scolastico, si è inoltre cercato di sviluppare le specifiche capacità e vocazioni dei membri delle comunità, anche attraverso progetti di auto-imprenditorialità.

Questo insieme di politiche deve essere integrato. La varietà e il coordinamento degli strumenti obbligano infatti a contrastare la reificazione di questo universo eterogeneo di minoranze in un'unica categoria amministrativa assegnata a un solo strumento di intervento (il campo "nomadi"), implicano un positivo superamento del trattamento differenziale e delle politiche specialistiche rivolte esclusivamente alle comunità zigane, che sono in realtà disfunzionali, al di là delle stesse intenzioni dei promotori, a quella conciliazione tra la specificità di una cultura e di una condizione sociale e i criteri universalistici di accesso e di fruizione dei servizi pubblici, contribuendo a rafforzare ulteriormente gli stereotipi assistenzialistici nei confronti di rom e sinti. Ancora, la varietà degli strumenti favorisce la predisposizione di interventi inter-scalari che riguardino tanto le comunità nel loro complesso - il cui coinvolgimento è necessario soprattutto in fase di progettazione, così come nei casi di conflitto con la società maggioritaria è necessario il coinvolgimento degli attori ostili alla presenza zigana e la mediazione delle esigenze e dei bisogni da essi espressi -, che le sin- 
gole famiglie e i signoli individui. Gli interventi di politica pubblica devono infatti accordarsi a tutti i livelli dell'esprienza sociale di rom e sinti, partendo nel corso dei progetti dal nucleo più grande (la comunità) per arrivare progressivamente a percorsi quasi-individuali.

Infine, la varietà e il coordinamento degli strumenti stimola i diversi settori dell'amministrazione a predisporre quei dispositivi di coordinamento istituzionale cui si è fatto più volte riferimento, all'interno dei quali i diversi settori dell'ente locale si coordinino tra loro e mettano in comunicazione l'ente locale con altre istituzioni e con i mediatori e i destinatari dell'azione pubblica. Tali dispositivi, e gli attori che ne fanno parte, possono svolgere un ruolo fondamentale sia rispetto alla reciproca traduzione dei rispettivi mondi culturali e delle pratiche sociali e organizzative tra comunità zigane e società maggioritaria, sia nella mediazione di casi di conflittualità.

\section{8. (False) Controversie in vista della programmazione di interventi}

Parte della letteratura sulle politiche per rom e sinti e, ancor più, attori del sistema politico e del sistema mediatico, disegnano tali politiche come un campo caratterizzato da un sistema di dicotomie: sostegno alla scolarizzazione diffusa $o$ scolarizzazione dell'élite; migranti o immigrati; interventi per i rom o interventi dei rom; finanziamento a progetti o formazione su programmazione e costruzione di consenso; sicurezza o politiche sociali; interlocuzione con i comuni o con le Provincie; un bando per i rom o quote per i rom dentro i bandi esistenti.

Le considerazioni svolte finora ci consentono di affermare che si tratti di false dicotomie. I progetti di inserimento scolastico che si sono rivelati maggiormente efficaci si pongono obiettivi di scolarizzazione diffusa, ma può essere funzionale al raggiungimento di tale obiettivo un'azione mirata specificamente alle élite delle comunità, che possa funzionare da esempio e garantire legittimazione ai progetti presso la comunità. L'importante è che comunque i progetti non puntino a creare tensioni fra i minori e i loro genitori, ma si rivolgano congiuntamente a entrambe le parti.

Rispetto alla dicotomia migranti/immigrati, abbiamo potuto osservare la radicale etereogenità della condizione giuridica e dello status di rom e sinti. Alcune situazioni - di privazione, di marginalità, di criminalità e microcriminalità - sono specificamente connesse a gruppi di recente immigrazione, quindi alla condizione e all'insieme di problematiche legate alla condizione di migranti. Ma vi è anche una forte componente di immigrati di seconda e terza generazione, la cui condizione non è accostabile alla precedente. È importante quindi discernere di volta in volta, nella progettazione degli interventi, se e in che misura specifiche situazioni siano legate a una condizione di recente immigrazione o a fattori più sedimentati e di lungo periodo, e quale sia la composizione delle comunità rispetto a queste variabili. 
Per quanto riguarda la dicotomia interventi dei/per i rom, abbiamo visto che non si dà una cosa senza l'altra: ogni intervento efficace per i rom è un intervento con i rom, già a partire dalla fase di progettazione.

Allo stesso modo, non sono - non dovrebbero essere - alternativi tra loro il finanziamento di progetti con obiettivi mirati e il tempo lungo della programmazione, che preveda importanti investimenti nella formazione di personale specializzato e una faticosa opera di costruzione del consenso al'interno della società locale. Ogni progetto finanziato acquista efficacia dal fatto di essere una parte, un momento, di un lavoro di programmazione e di ricerca del consenso di medio-lungo termine. Un consenso da costruire in modo «incrementale», da un lato coinvolgendo attivamente i diversi segmenti della società locale interessati direttamente o indirettamente alla realizzazione di politiche per rom e sinti (anche quelli più ostili), dall'altro fornendo alla stessa società locale prove di realtà che le consentano di osservare il concreto raggiungimento di obiettivi anche parziali rispetto all'integrazione e alla riduzione della marginalità e della privazione di queste minoranze.

È evidente la correlazione tra sicurezza e politiche sociali. L'efficacia delle seconde retroagisce positivamente sulla prima e costituisce una prova di realtà che consente di allargare il consenso sulle politiche di integrazione. Ciò non significa che vadano sottaciute difficoltà specifiche legate a problemi di sicurezza, crminalità e microcriminalità all'interno delle comunità. È noto infatti che come in tutte le situazioni sociali caratterizzate da un basso livello di integrazione, da povertà, marginalità e precarietà delle condizioni di vita - situazioni di criminalità sono presenti all'interno delle comunità rom e sinti (cfr, paragrafo 4.1.). Probelmi da tenere senz'altro presenti e da affrontare, congiuntamente alle istituzioni pubbliche, con gli strumenti odinari (e non con strumenti emergenziali e straordinari) delle politiche di sicurezza e del diritto penale, ma che vanno al contempo aggrediti agendo proprio su integrazione e riduzione della marginalità attraverso le politiche sociali.

Per quanto riguarda le ultime due false dicotomie che indicavamo, abbiamo già osservato come i progetti e le politiche devono coinvolgere le istituzioni in una dimensione multilivello, che coinvolga tanto il livello comunale che quello provinciale e, possibilmente, regionale. All'interno dei Comuni, centrale è l'attribuzione di responsabilità a specifiche strutture amministrative - assessorati, uffici comunali - che possano grantire alle politiche e ai progetti una continuità nel tempo che trascenda le fluttuazioni a cui li sottopone il ciclo politico amministrativo di cinque anni, e che possano quindi portarle avanti al di là della contingenza politica. Per non alimentare politiche e atteggiamenti culturali di tipo differenzialista, infine, seppure non si possa escludere di orientarsi in determinate situazioni a bandi pubblici specificamente indirizzati a rom e sinti, maggiormente efficaci si sono finora rivelate politiche in cui ad essi sono riservate delle quote all'interno di bando rivolti ad una popolazione più ampia. 


\section{Bibliografia}

Alietti A., 2009, "Generazioni nomadi tra tradizione e mutamento", in Vitale T. (a cura di), Politiche possibili. Abitare le città con i rom e i sinti, Roma, Carocci.

Bonetti P., I nodi giuridici della condizione di rom e sinti in Italia", in Bonetti P., Simoni A., Vitale T. (a cura di), La Condizione Giuridica Di Rom e Sinti In Italia, Giuffré, Milano, 2011.

Marinaro I., Integration or marginalization? The failures of social policy for the Roma in Rome, Modern Italy, 8 (2), pp. 203-218, 2003.

Piasere L., Rom, sinti e camminanti nelle scuole italiane, in Gobbo F. ( a cura di), "Processi educativi nelle società multiculturali”, CISU, Roma, 2007, pp. 161-172.

Sacco A., La scuola. Luogo di appartenenza o di esclusione? in Vitale T. (a cura di ), Politiche possibili. Abitare le città con i rom e $i$ sinti, Carocci, Roma, 2009.

Tosi A., Cagnoli R., Pessina G., Un'indagine sulla presenza di rom e sinti in Lombarda: primi risultati, in Ambrosini M., Tosi A. (a cura di), Vivere ai margini: un'indagine sugli insediamenti rom e sinti in Lombardia, Ismu, Milano, 2007.

Vitale T., Politica e politiche fanno la differenza, Il Regno, 22, 2010.

Vitale T., Arrigoni P., Quale legalità? Rom e gagi a confronto, Aggiornamenti sociali, 3, 2008, pp. 182-194.

Vitale T., Claps E., Arrigoni P., Regards croisés. Antitsiganisme et possibilité du vivre ensemble, Roms et gadjés, en Italie. Etudes tsiganes, 35, 2009, pp. 80-103.

Vitale T., Claps E., Arrigoni P., I sondaggi e il loro uso. Problemi di cecità logica a partire dal caso dei Rom, in Comunicazione Politica (Com Pol), n. 2/2011, pp. 167-95. 